\title{
Influence of Student Depression on the Spread of Public Opinion in University
}

\author{
Nie Min, Yang Lei, Luo Weimin, Guowu Yang, and Hu Xia $i$ \\ Big Data Research Center, University of Electronic Science and Technology of China, Chengdu 610054, China \\ Correspondence should be addressed to Hu Xia; xiahu@uestc.edu.cn
}

Received 2 June 2020; Revised 29 June 2020; Accepted 6 July 2020; Published 23 September 2020

Guest Editor: Qingyi Zhu

Copyright ( $) 2020$ Nie Min et al. This is an open access article distributed under the Creative Commons Attribution License, which permits unrestricted use, distribution, and reproduction in any medium, provided the original work is properly cited.

\begin{abstract}
In this study, we examined the impact of college students' mental health on their social behavior. A social network was identified based on the behavior of college students sharing a meal. We analyzed the impact of depression on the structure of this network and found that students without obvious depressive symptoms, based on the test data of the SCL-90 Assessment Scale, were better at socializing than students with obvious depressive symptoms. We proposed a public opinion spreading model on social networks and formulated a heterogeneous mean-field theory to describe it. Further, using computer simulation experiments, we studied the impact of students' mental health on the process of information propagation in college. The results of the experiments showed that students without obvious depressive symptoms were more likely to receive information than students with obvious depressive symptoms. Based on the results of our study, college psychological consultants can actively identify students who may be at risk of mental illness and give them attention and guidance.
\end{abstract}

\section{Introduction}

In the present self-media era, people can communicate online with friends through various platforms such as WeChat, QQ, Weibo, and BBS [1-4]. College students are interested in novelty and are the main users of various social platforms. On the one hand, students can communicate through these platforms to improve their studies. On the other hand, various views and opinions held by disgruntled members of colleges and universities also pass through these social platforms as public opinion and have a significantly negative impact on students. Therefore, understanding the principles and mechanisms of public opinion dissemination in colleges and universities has become a focus of intensive research study. The core of these studies lay in analyzing information dissemination on social networks [5-12].

Structurally, online social networks often have characteristics such as small-world and scale-free properties and high clustering coefficient [13-17]. These characteristics have a strong influence on the information propagation process, propagation range, and burst threshold [18-23]. Watts [24], a well-known network science scholar, analyzed the spread of news stories, videos, and pictures on Twitter and found that the propagation process showed a substantial structural diversity; "hot" events, which spread quickly over the social network, often have both viral propagation and broadcast characteristics. Lu and Zhou [4] found that it was easier to spread information on high-cluster coefficient networks. For high-risk information (such as political information), single dissemination cannot eliminate the risks and uncertainties caused by the dissemination of the information; multiple confirmations become particularly important $[25,26]$. Zheng et al. [27] proposed complex propagation dynamics based on multiple confirmations to characterize the information propagation process in social networks. It was found that the smaller the average, the higher the difference in the spread of information on regular and random networks. Because of the limited risk of redundant information to eliminate the propagation [28], $\mathrm{Wu}$ et al. [29-31] further analyzed information propagation on multilayered social networks based on the theory of edge division [32]. During the dissemination process, it was found that the multilayer network structure is comparatively more conducive to information dissemination. Shu et al. [33] 
found that the interlayer network structure is particularly crucial to information spread.

In the dissemination of public opinion in colleges and universities, different students have different roles. For example, students who frequent the library may have short online communication times. By contrast, students who live in dormitories may have an extended online learning and communication time, which leads to differences in the influence of different student attributes on colleges' public opinion communication attributes. In this study, we constructed a public opinion spreading model to determine whether students with apparent symptoms of depression influence the spread of public opinion in colleges and universities.

\section{Data Descriptions}

To quantify the impact of college students' mental health on their social relationships in school, we collected data from 4955 freshmen in a college, including the SCL-90 Assessment Scale (hereafter referred to as the SCL-90 form) of 20188 swipe-card data in October 21-31, 2018. The data used in the article, which could only be used for statistical analysis to obtain certain macrostatistical characteristics, were all processed through anonymization; hence, it is impossible to identify specific students. Derogatis proposed the SCL-90 table in 1975. The table is deployed for comprehensively scoring people's mental health on multiple levels, such as feelings, emotions, thoughts, consciousness, behavior, lifestyle, interpersonal relationships, diet, and sleep, and quantifying multiple depression. Related test indicators include somatization, obsessive-compulsive symptoms, interpersonal sensitivity, depression, anxiety, hostility, terror, paranoia, and psychosis. Based on the respondent's answer, the degree of depressive symptoms is established on a scale of 1 to 5, which indicates "none," "very light," "moderate," "heavy," and "serious." The overall score range of the respondents was [13-65]. Based on their scores, respondents can be divided into five categories: none (no symptoms), mild (symptomatic but infrequent), moderate (symptomatic and frequent), heavy (symptomatic and severe), and severe (symptomatic and very serious). To simplify the research, in this follow-up study, the subjects were divided into two categories based on whether they had or did not have obvious depressive symptoms. The overall score range of students without apparent symptoms of depression was [13-26]. Students with moderate, heavy, and severe symptoms, with an overall score range of [27-65], were categorized as those with apparent symptoms of depression.

Among the 4955 students, 3879 were male and 1076 were female. The minimum age was 16.1 years (as of October 1, 2018 ), the maximum was 25.1 , the average was 18.5 , and the median was 18.4. Dining in the cafeteria is a typical behavior of college students that can reflect students' social behavior to a certain extent. Therefore, we built a social network using the swiping-card data from the student canteen. The graph $G(V, E)$ was used to represent a social network, where $V$ represents a set of nodes and $E$ represents a set of connected edges. The nodes represented students, with the edges representing the social relationships between them. We inferred that there was a link between two students that met the following three conditions at the same time: (1) they spent a similar length of time on a credit card machine; (2) their spending time interval was less than two minutes; (3) During the entire observation time (August 21 to October 31, 2018), the number of times conditions (1) and (2) were met was greater than the threshold $T$.

\section{Propagation Model Description}

The college students' social network $G$ could further determine whether the students had obvious symptoms of depression. Students with obvious depressive symptoms were recorded as $G_{H}$, and those without obvious depressive symptoms were recorded as $G_{N}$. Mathematically, the social network $G$ and the network $W$ are adjacent weights; $w_{i j}=n_{i j}$ represents the number of meals the two categories of students have in a short period. The greater $w_{i j}$, the closer the relationship between student $i$ and student $j$.

To describe the propagation process of public opinion in universities, we proposed a generalized susceptible-infected-recovered (SIR) model. The susceptible students were those that did not know the news but were likely to come to know it. The infected students were those that already knew the news and were willing to share it with friends. The recovered students were those that were not interested in the information. At the initial moment, a student was randomly selected as infected. At a specific moment $t$, there was a certain probability of each infected student $i$ informing a susceptible neighbor. To consider the spread of student depression arising from public opinion information in universities, we assumed that the probability of students passing information to students with depression and those without depression was heterogeneous and could be specifically divided into the following four cases:

(i) If the student $i$ had no obvious depressive symptoms, the probability that he would pass the information to a friend without obvious depressive symptoms $j$ was $\lambda_{\mathrm{NN}}$, where $N$ indicates no obvious symptoms of depression. Specifically, $\lambda_{\mathrm{NN}}$ is expressed as follows:

$$
\lambda_{\mathrm{NN}}=1-\left(1-\lambda_{\mathrm{nn}}\right)^{w_{i j}}
$$

(ii) If the student $i$ had no obvious symptoms of depression, the probability that he would pass the information to a friend with obvious symptoms of depression $j$ was $\lambda_{\mathrm{NH}}$, where the subscript $H$ indicates the presence of obvious symptoms of depression. Similarly, $\lambda_{\mathrm{NH}}$ is expressed as follows:

$$
\lambda_{\mathrm{NH}}=1-\left(1-\lambda_{\mathrm{nh}}\right)^{w_{i j}}
$$

(iiii) If the student $i$ had obvious depressive symptoms, the probability that he would pass the information 
to a friend without obvious depressive symptoms $j$ was

$$
\lambda_{\mathrm{HN}}=1-\left(1-\lambda_{\mathrm{hn}}\right)^{w_{i j}}
$$

(iv) If the student $i$ had no obvious symptoms of depression, the probability that he would pass the information to a friend with obvious symptoms of depression $j$ was

$$
\lambda_{\mathrm{HH}}=1-\left(1-\lambda_{\mathrm{hh}}\right)^{w_{i j}}
$$

Students' depression has different effects on the transmission of different types of information. The probability of each student losing interest in the news was $\gamma$. The propagation dynamics evolved until there were no infected students in the system.

\section{Theoretical Analysis}

In this section, we propose a set of heterogeneous mean-field theories to characterize the public opinion propagation model. The degree of distribution of the network $G$ is denoted as $P\left(k_{H}, k_{N}\right)$, which indicates that a randomly selected node $i$ has $k_{H}$ friends with obvious depressive symptoms and $k_{N}$ friends with no obvious depressive symptoms. $S_{X}\left(k_{H}, k_{N}, t\right), \rho_{X}\left(k_{H}, k_{N}, t\right)$, and $R_{X}\left(k_{H}, k_{N}, t\right)$ were used to indicate that students with degree $\left(k_{H}, k_{N}\right)$ were in the susceptible, infected, and recovered states, respectively, where $X \in\{H, N\}$ indicates whether the student had obvious symptoms of depression. At the initial time, that is, $t=0$, we have $S\left(k_{H}, k_{N}, t\right)=(1-1 / n)$, $\rho\left(k_{H}, k_{N}, t\right)=(1 / n)$, and $R\left(k_{H}, k_{N}, t\right)=0$, where $n$ represents the number of nodes in the network $G$.

A student $i_{H}$ with obvious depressive symptoms with degree $\left(k_{H}, k_{N}\right)$ is in a susceptible state and needs to satisfy two conditions at the same time: (1) he is not infected by a friend with obvious symptoms and (2) he is not infected by a friend without obvious symptoms. For condition (1), the probability that the student $i_{H}$ is connected to an infected friend with obvious symptoms is

$$
c_{\mathrm{HH}}=\frac{\sum_{k_{H}^{\prime}} P\left(k_{H}^{\prime}, K_{N}^{\prime}\right) k_{H}^{\prime} \rho_{H}\left(k_{H}^{\prime}, K_{N}^{\prime}\right)}{\left\langle k_{H}\right\rangle} .
$$

where $\left\langle k_{H}\right\rangle$ indicates the number of students with obvious depressive symptoms. Therefore, the probability of the student $i_{H}$ not getting infected by a friend with obvious depressive symptoms is

$$
\eta_{\mathrm{HH}}(t)=1-S_{H}\left(k_{H}, k_{N}, t\right) \lambda_{\mathrm{HH}} c_{\mathrm{HH}} \cdot
$$

Similarly, we can obtain the probability of satisfying condition (2), that is, the probability of the student $i_{H}$ not being infected by a friend without obvious depressive symptoms, as follows:

$$
\eta_{\mathrm{HN}}(t)=1-S_{H}\left(k_{H}, k_{N}, t\right) \lambda_{\mathrm{HN}} c_{\mathrm{HN}},
$$

where $c_{\mathrm{HN}}$ represents the probability of the student $i_{H}$ being connected to an infectious friend without obvious symptoms, which is expressed as follows:

$$
c_{\mathrm{HN}}=\frac{\sum_{k_{N}^{\prime}} P\left(k_{H}^{\prime}, K_{N}^{\prime}\right) k_{H}^{\prime} \rho_{H}\left(k_{H}^{\prime}, K_{N}^{\prime}\right)}{\left\langle k_{\mathrm{HN}}\right\rangle},
$$

where $\left\langle k_{\mathrm{HN}}\right\rangle$ represents the number of students with obvious depressive symptoms who have no obvious depressive symptoms.

Considering conditions (1) and (2) comprehensively, the probability of student $i_{H}$ being infected at $t$ was

$$
\chi_{H}(t)=1-\left(1-\eta_{\mathrm{HH}}(t)\right)\left(1-\eta_{\mathrm{HN}}(t)\right) .
$$

Therefore, we can obtain the evolution equation of $S_{H}\left(k_{H}, k_{N}, t\right)$ as

$$
\frac{\mathrm{d} S_{H}\left(k_{H}, k_{N}, t\right)}{\mathrm{d} t}=-\chi_{H}(t)
$$

For those infected with obvious depressive symptoms, this growth stems from the infection of the susceptible nodes by neighbors, and the decrease comes from recovery. Therefore, the state evolution equation of $\rho_{H}\left(k_{H}, k_{N}, t\right)$ is

$$
\frac{\mathrm{d} \rho_{H}\left(k_{H}, k_{N}, t\right)}{\mathrm{d} t}=\chi_{H}(t)-\gamma \rho_{H}\left(k_{H}, k_{N}, t\right) .
$$

The state evolution equation of $R_{H}\left(k_{H}, k_{N}, t\right)$ is

$$
\frac{\mathrm{d} R_{H}\left(k_{H}, k_{N}, t\right)}{\mathrm{d} t}=\gamma \rho_{H}\left(k_{H}, k_{N}, t\right) .
$$

Similar to the aforementioned discussion, it can be observed that the degree of the nodes indicating students without obvious depressive symptoms is $\left(k_{H}, k_{N}\right)$; the evolution equations in the susceptible, infected, and recovered states, respectively, were

$$
\begin{aligned}
& \frac{\mathrm{d} S_{N}\left(k_{H}, k_{N}, t\right)}{\mathrm{d} t}=-\chi_{N}(t), \\
& \frac{\mathrm{d} \rho_{N}\left(k_{H}, k_{N}, t\right)}{\mathrm{d} t}=\chi_{N}(t)-\gamma \rho_{N}\left(k_{H}, k_{N}, t\right),
\end{aligned}
$$

with

$$
\frac{\mathrm{d} R_{N}\left(k_{H}, k_{N}, t\right)}{\mathrm{d} t}=\gamma \rho_{N}\left(k_{H}, k_{N}, t\right),
$$

where

$$
\chi_{N}(t)=1-\left(1-\eta_{\mathrm{NN}}(t)\right)\left(1-\eta_{\mathrm{NH}}(t)\right)
$$

The probability of a student without obvious depressive symptoms $i_{N}$ connecting to an infected friend without obvious symptoms was

$$
c_{\mathrm{NN}}=\frac{\sum_{k_{N}^{\prime}} P\left(k_{H}^{\prime}, K_{N}^{\prime}\right) k_{H}^{\prime} \rho_{N}\left(k_{H}^{\prime}, K_{N}^{\prime}\right)}{\left\langle k_{N}\right\rangle} .
$$


where $\left\langle k_{N}\right\rangle$ represents the number of students with no obvious depressive symptoms connected to those without obvious depressive symptoms and $c_{\mathrm{NH}}$ indicates the probability of the student $i_{N}$ connecting to an infected friend with obvious symptoms, which is expressed as follows:

$$
c_{\mathrm{NH}}=\frac{\sum_{k_{H}^{\prime}} P\left(k_{H}^{\prime}, K_{N}^{\prime}\right) k_{H}^{\prime} \rho_{H}\left(k_{H}^{\prime}, K_{N}^{\prime}\right)}{\left\langle k_{\mathrm{NH}}\right\rangle} .
$$

where $\left\langle k_{\mathrm{NH}}\right\rangle$ represents the ratio of students with obvious depressive symptoms to those without obvious depressive symptoms.

We have presented the evolution equations of students with and without obvious depressive symptoms in various states. It is not difficult to obtain the proportion of various nodes in the SIR states in this system, as follows:

$$
\begin{aligned}
& S(t)=\sum_{k_{H}, k_{N}} P\left(k_{H}, k_{N}\right)\left[S_{H}\left(k_{H}, k_{N}, t\right)+S_{N}\left(k_{H}, k_{N}, t\right)\right], \\
& \rho(t)=\sum_{k_{H}, k_{N}} P\left(k_{H}, k_{N}\right)\left[\rho_{H}\left(k_{H}, k_{N}, t\right)+\rho_{N}\left(k_{H}, k_{N}, t\right)\right], \\
& R(t)=\sum_{k_{H}, k_{N}} P\left(k_{H}, k_{N}\right)\left[R_{H}\left(k_{H}, k_{N}, t\right)+R_{N}\left(k_{H}, k_{N}, t\right)\right],
\end{aligned}
$$

respectively.

Next, we determined the outbreak threshold of public opinion. At $t \longrightarrow 0$, only a few nodes in the network were informed; consequently, $\rho_{H}(t) \longrightarrow 0$, and $\rho_{N}(t) \longrightarrow 0$. Therefore, we can obtain the Jacobian matrix of the equations (11) and (14) as $H$. The dimension of the matrix $H$ is $k_{H}^{\max } k_{N}^{\max } \times k_{H}^{\max } k_{N}^{\max }$. The critical condition to be met by the outbreak threshold is as follows:

$$
\lambda_{c}=\frac{1}{\Lambda(H)},
$$

where $\Lambda(H)$ represents the maximum eigenvalue of the matrix $H$.

4.1. Numerical Simulation. In this section, we applied the public opinion information dissemination model on the real-world social network and randomized network and analyzed the influence of the network structure on information dissemination. We set the threshold as $T=2$. The randomized null model was constructed as follows. (1) Two connected edges, $e_{1}$ and $e_{2}$, were randomly selected. $e_{1}$ was connected to nodes $i$ and $j$, and $e_{2}$ was connected to the nodes $m$ and $n$. If the network does not have double edges and self-loops, when the nodes connected by the two connected edges are exchanged, then the nodes were exchanged; otherwise, the nodes were left as they were. (2) Step (1) was repeated until the $10 E$ step, where $E$ represents the number of edges.

In the experimental simulation process, we calculated the probability of propagating information between nodes of the same type as equal, denoted as $\lambda_{\text {in }}$; the probability of propagation between nodes of different types was equal, denoted as $\lambda_{\text {between }}$. Using the same parameters, we conducted, at least, 1000 experimental simulations to calculate the average values.

First, we analyzed the network topology of the social network. In Figure 1(a), the relationship between students without obvious depressive symptoms is shown. Compared with the zero model of stochastic networks, large-degree nodes in real networks tend to connect with small-degree nodes. In Figure 1(b), we also observed a similar phenomenon in the context of a meal-sharing social network. Only a few large-degree nodes dined together; the connection was more of a star-shaped knot, that is, large-degree nodes were connected to more small-degree nodes.

Figure 2 shows the clustering coefficients of students with or without obvious depressive symptoms. We found that the clustering coefficient $c_{k}$ decreased with $k$, regardless of whether the students had obvious depressive symptoms. Compared to the null model, when $k \leq 400$, the students had a high clustering coefficient, which indicated that nodes with a small degree were more inclined to have a group meal with three people, while nodes with a high degree had few meals.

In Figure 3, we show the proportion of susceptible, infectious, and recovered states over time of students with and without obvious depressive symptoms. In the figure, $\lambda_{\text {in }}=0.004, \lambda_{\text {between }}=0.006$, and the recovery probability $\gamma=0.1$. For students without obvious depressive symptoms, we found that the real network was not conducive to information dissemination. This phenomenon may be attributable to the following reasons. Compared with the random network, the real network has a higher cluster coefficient. When the information dissemination rate is high, information in the network with a high clustering coefficient always passes to the surrounding nodes, and as it is not easy to reach nodes beyond the surrounding ones, this environment is not conducive to information dissemination. Similarly, among students without obvious depressive symptoms, it was more difficult to disseminate information on the real network compared to the random ones, as shown in Figure 3(b).

In Figure 4, we present a graph of the evolution of the state of the nodes in the network over time. Figures 4(a)-4(c) show that $t=0, t=5$, and $t=t_{\max }$ on the random network in Figure 3. Students with significant depressive symptoms were infected. In the figure, green indicates the susceptible state, red indicates the infected state, and blue indicates the recovered state. Similarly, Figures $4(\mathrm{~d})-4(\mathrm{f})$ show students without obvious depressive symptoms on random networks at $t=0, t=5$, and $t=t_{\max }$. Figures $4(\mathrm{~g})-4(\mathrm{i})$ show students with obvious depressive symptoms on the real network, when $t=0, t=5$, and $t=t_{\max }$. Figures $4(\mathrm{j})-4(\mathrm{l})$ show the state of students who have no obvious depressive symptoms on the real network at time $t=0, t=5$, and $t=t_{\text {max }}$.

In Figure 5, we show the spread of information among students on real social networks in detail. In the figure, we set the recovery probability to $\gamma=1.0$. Through numerous experimental simulations, we found that varying the value of $\gamma$ did not qualitatively change the propagation phenomenon. Figure 5(a) shows the proportion of students without significant depressive symptoms receiving information $R_{N}$ as a 


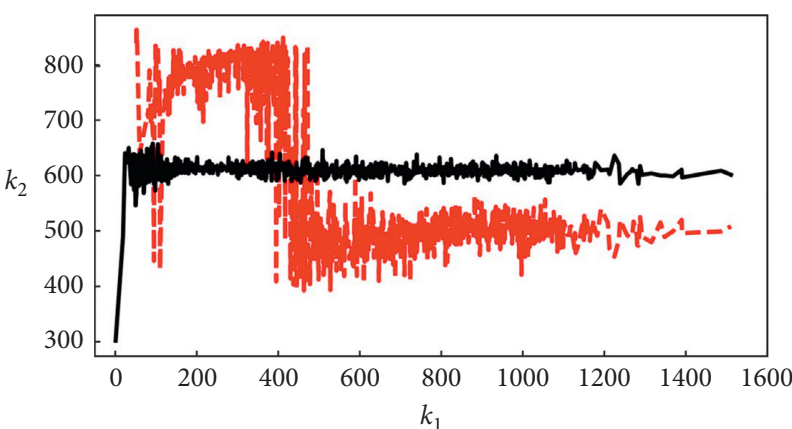

- - - Real data

— Null model

(a)

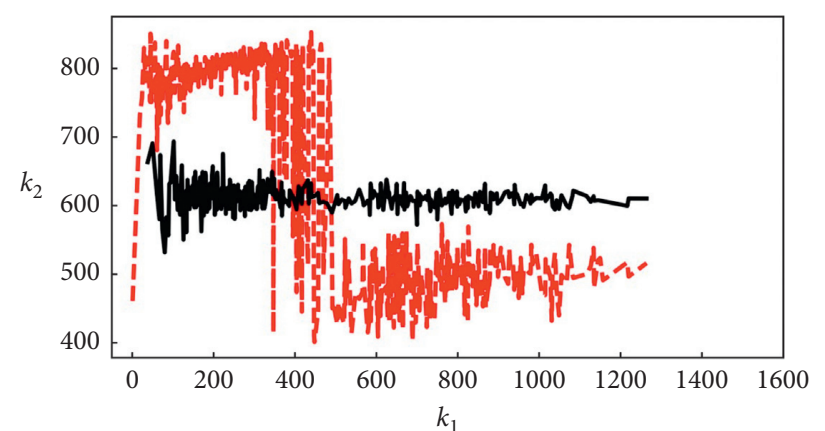

- - - Real data

— Null model

Figure 1: Degree correlation for (a) real-world network and (b) null model network.



(a)



(b)

FIGURE 2: Clustering for (a) real-world network and (b) null model network.


(a)



$\square \rho_{H}(t)$, real-world network $\square-\rho_{H}(t)$, null model network
$\square R_{H}(t)$, real-world network $\square R_{H}(t)$, null model network
$\square S_{H}(t)$, real-world network $\square S_{H}(t)$, null model network

(b)

FIgURE 3: The status of various students evolves over time on social network (a) with and (b) without obvious depressive symptoms. 


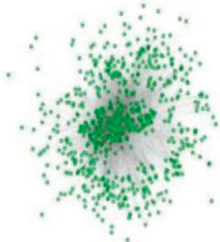

(a)

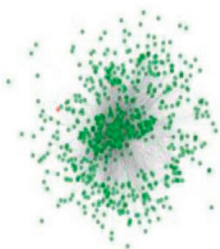

(g)



(b)

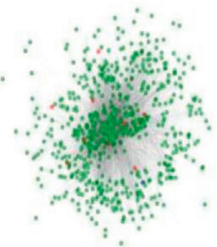

(h)

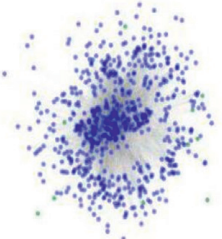

(c)

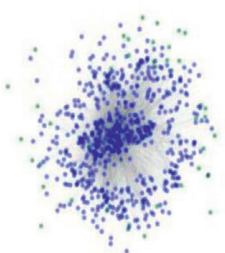

(i)



(d)

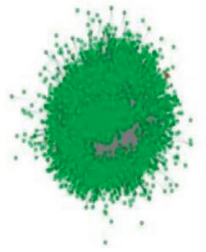

(j)



(e)

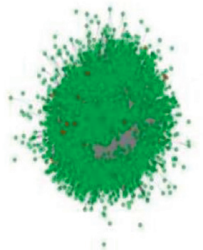

(k)

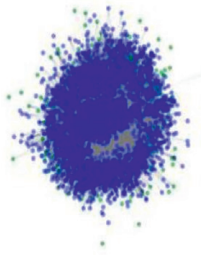

(f)

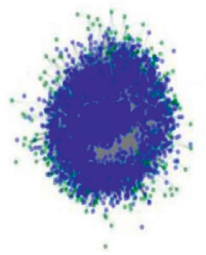

(1)

Figure 4: Node state on different time steps.



(a)



(b)



(c)

FIGURE 5: Information spreading on real-world networks.

function of $\lambda_{\text {in }}$ and $\lambda_{\text {between }}$. As $\lambda_{\text {in }}$ and $\lambda_{\text {between }}$ increased, $R_{N}$ also increased. When both $\lambda_{\text {in }}$ and $\lambda_{\text {between }}$ were less than their outbreak threshold (can be predicted by using equation (21)), it was almost impossible for students without obvious symptoms of depression to receive information. When $\lambda_{\text {in }}$ or $\lambda_{\text {between }}$ was greater than the outbreak threshold, a large number of students without obvious depressive symptoms could receive the message. When $\lambda_{\text {in }}\left(\lambda_{\text {between }}\right)$ was sufficiently large, $\lambda_{\text {between }}=0\left(\lambda_{\text {in }}=0\right)$ could cause global information bursts. We observed a similar phenomenon in Figures 5(b) and 5(c), which show $R_{H}$ and $R$ with $\lambda_{\text {in }}$ and $\lambda_{\text {between }}$, respectively.
Figure 6 shows the spread of public opinion on the corresponding null model random network. Among them, the three subgraphs show the changes in $R_{N}, R_{H}$, and $R$ with $\lambda_{\text {in }}$ and $\lambda_{\text {between }}$. From the three figures, a phenomenon similar to Figure 3 can be observed.

To further compare the impact of the real network structure on information dissemination, we show the difference in the spread of information on real and random networks in Figure 7. For students without obvious depressive symptoms, the difference in the spread was defined as

$$
\Delta R_{N}=R_{N}^{\text {real }}-R_{N}^{\text {random }}
$$




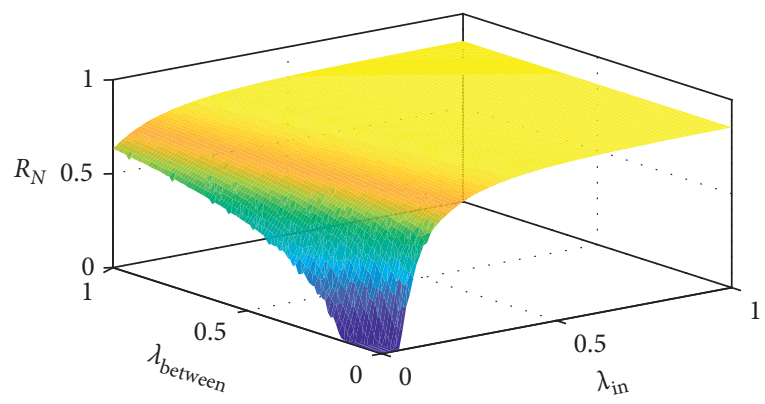

(a)

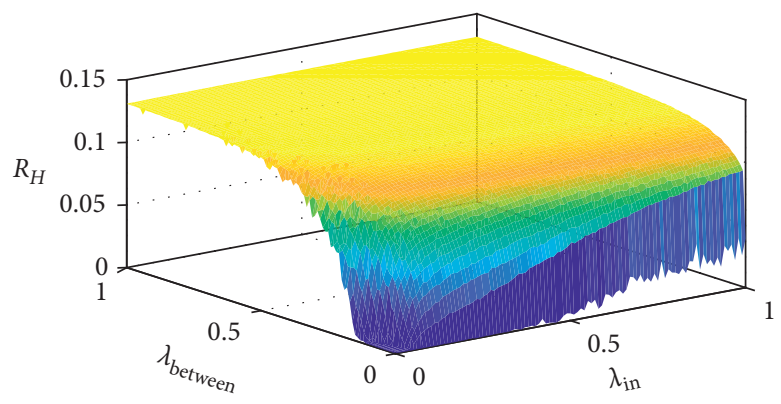

(b)

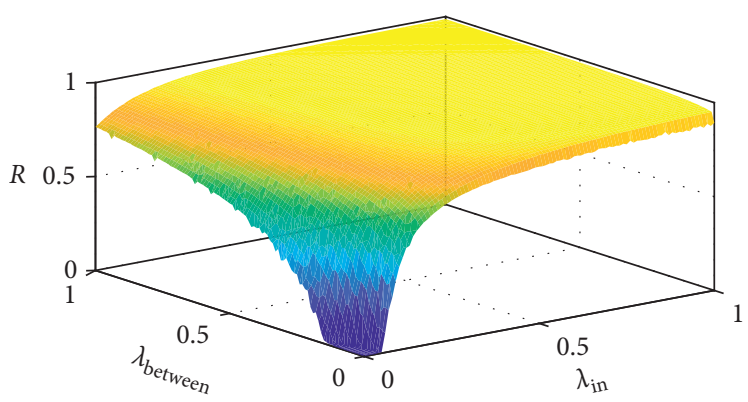

(c)

FIGURE 6: Information spreading on null model networks.

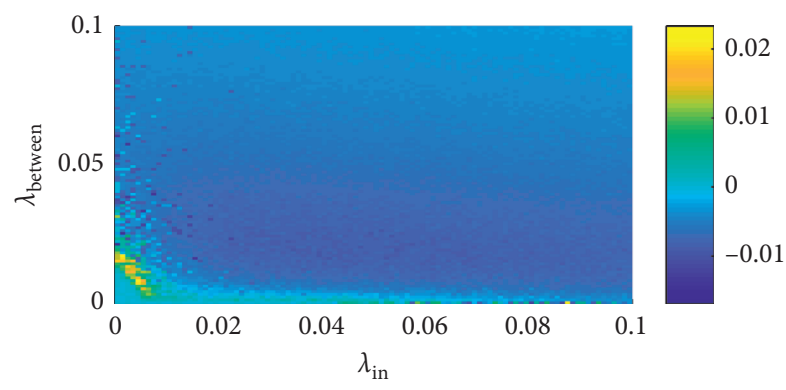

(a)

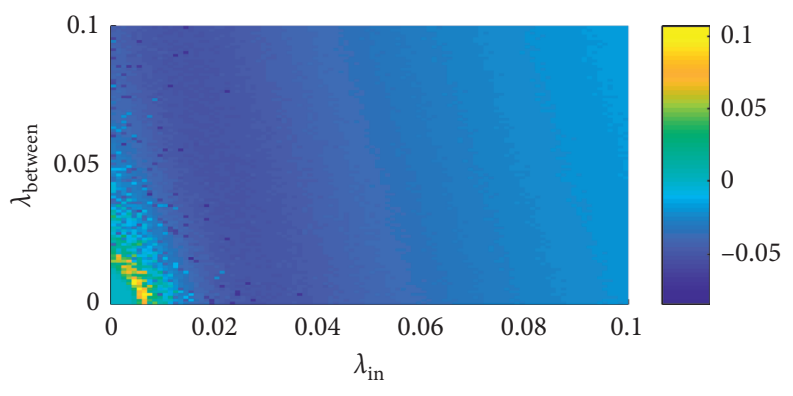

(b)

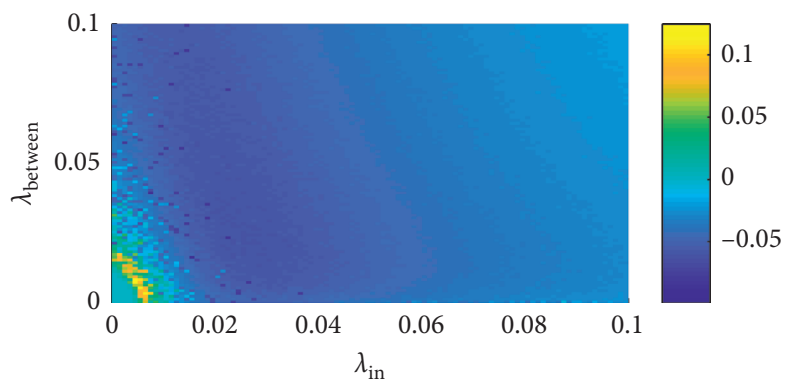

(c)

FIGURE 7: Difference of information spreading on real-world and null model networks.

where $R_{N}^{\text {real }}$ and $R_{N}^{\text {random }}$ represent the spread of information on real and random networks, respectively. Similarly, the difference in the spread of the proportion of students with significant depressive symptoms receiving information and the proportion of all students receiving information was $\Delta R_{H}$ and $\Delta R$. Figures $7(\mathrm{a})-7$ (c) show $\Delta R_{N}$, $\Delta R_{H}$, and $\Delta R$ along with $\lambda_{\text {in }}$ and $\lambda_{\text {between }}$ changes. We found that when $\lambda_{\text {in }}$ and $\lambda_{\text {between }}$ were small, $\Delta R_{N}, \Delta R_{H}$, and $\Delta R$ were all greater than zero, which indicates that the true network structure was not conducive to information dissemination. Because the real network had a high clustering coefficient, it was conducive to the spread of information in a local range. With increase in $\lambda_{\text {in }}$ and $\lambda_{\text {between }}, \Delta R_{N}, \Delta R_{H}$, and $\Delta R$ were all less than zero, that is, 
the real network was not conducive to information dissemination.

\section{Conclusions}

We proposed a model of public opinion information dissemination based on the SIR model. In the model, the students were divided into two categories based on whether they had obvious depressive symptoms or not. We proposed a heterogeneous mean-field theory to characterize the model. Several computer simulations revealed that students without obvious depressive symptoms were more likely to receive information than students with obvious depressive symptoms. Further, due to the existence of high clustering coefficients in real social networks, there were two scenarios of information propagation. When the information dissemination rate was low, it was easier to disseminate information on real social networks than on random networks. The results help us to have a deeper understanding of the effects of student depression on the spread of public opinion. In big data era, the online data about the student activity should be taken into consideration when building their social network and the effects of student depression on the spread of public opinion in university should be further investigated.

\section{Data Availability}

No data were used to support this study.

\section{Conflicts of Interest}

The authors declare that they have no conflicts of interest.

\section{References}

[1] S. Pei and H. A. Makse, "Spreading dynamics in complex networks," Journal of Statistical Mechanics: Theory and Experiment, vol. 2013, no. 12, p. P12002, 2013.

[2] T. R. Zaman, R. Herbrich, J. Van Gael, and D. Stern, "Social media's perspective on industry 4.0: a twitter analysis," in Proceedings of the Workshop on Computational Social Science and the Wisdom of Crowds, Whistler, Canada, December 2010.

[3] D. Wang, Z. Wen, H. Tong, C.-Y. Lin, C. Song, and A.-L. Barabási, "Information spreading in context," in Proceedings of the 20th International Conference on World Wide Web (2011), pp. 735-744, Hyderabad, India, March 2011.

[4] L. Lü and T. Zhou, "The small world yields the most effective information spreading," New Journal of Physics, vol. 13, no. 12, p. 123005, 2011.

[5] M. Zanin, D. Papo, P. A. Sousa et al., "Combining complex networks and data mining: why and how," Physics Reports, vol. 635 , pp. 1-44, 2016.

[6] A. L. Lloyd and R. M. May, "Epidemiology: how viruses spread among computers and people," Science, vol. 292, no. 5520, pp. 1316-1317, 2001.

[7] Z.-K. Zhang, C. Liu, X.-X. Zhan, X. Lu, C.-X. Zhang, and Y.-C. Zhang, "Dynamics of information diffusion and its applications on complex networks," Physics Reports, vol. 651, pp. 1-34, 2016.
[8] X.-X. Zhan, A. Hanjalic, and H. Wang, "Information diffusion backbones in temporal networks," Scientific Reports, vol. 9, p. 1, 2019.

[9] R. Pastor-Satorras and C. Castellano, "Distinct types of eigenvector localization in networks," Scientific Reports, vol. 6, p. 18847, 2016.

[10] Y. Moreno, J. B. Gómez, and A. F. Pacheco, "Epidemic incidence in correlated complex networks," Physical Review E, vol. 68, Article ID 035103, 2003.

[11] D. M. J. Lazer, M. A. Baum, Y. Benkler et al., "The science of fake news," Science, vol. 359, no. 6380, pp. 1094-1096, 2018.

[12] M. A. Porter and J. P. Gleeson, "Dynamical systems on networks," 2016, http://arxiv.org/abs/1403.7663.

[13] A.-L. Barabási and R. Albert, "Emergence of scaling in random networks," Science, vol. 286, no. 5439, pp. 509-512, 1999.

[14] M. Newman, Networks: An Introduction, Oxford University Press, Oxford, UK, 2010.

[15] A. Clauset, C. R. Shalizi, and M. E. J. Newman, "Power-law distributions in empirical data," SIAM Review, vol. 51, no. 4, pp. 661-703, 2009.

[16] S. Boccaletti, V. Latora, Y. Moreno, M. Chavez, and D. Hwang, "Complex networks: structure and dynamics," Physics Reports, vol. 424, no. 4-5, pp. 175-308, 2006.

[17] R. Cohen and S. Havlin, Complex Networks: Structure, Robustness and Function, Cambridge University Press, Cambridge, UK, 2010.

[18] Z. Wang, C. Xia, Z. Chen, and G. Chen, "Epidemic propagation with positive and negative preventive information in multiplex networks," IEEE Transactions on Cybernetics, vol. 99, 2020.

[19] Q. Shao, C. Xia, L. Wang, and H. Li, "A new propagation model coupling the offline and online social networks," Nonlinear Dynamics, vol. 98, no. 3, pp. 2171-2183, 2019.

[20] Z. Wang, Q. Guo, S. Sun, and C. Xia, "The impact of awareness diffusion on SIR-like epidemics in multiplex networks," Applied Mathematics and Computation, vol. 349, pp. 134-147, 2019.

[21] C. Gan, Q. Feng, Q. Zhu, Z. Zhang, Y. Zhang, and Y. Xiang, "Analysis of computer virus propagation behaviors over complex networks: a case study of oregon routing network," Nonlinear Dynamics, vol. 1, 2020.

[22] C. Gan, L. Wang, Z. Zhang, and Z. Wang, "Sparse attention based separable dilated convolutional neural network for targeted sentiment analysis," Knowledge-Based Systems, vol. 188, p. 104827, 2020.

[23] Y. Yi, Z. Zhang, L. T. Yang, C. Gan, X. Deng, and L. Yi, "Blockchain empowered cooperative authentication with data traceability in vehicular edge computing," IEEE Transactions on Network Science and Engineering, vol. 69, 2020.

[24] S. Goel, A. Anderson, J. Hofman, and D. J. Watts, "The structural virality of online diffusion," Management Science, vol. 62, p. 180, 2016.

[25] D. Centola, "An experimental study of homophily in the adoption of health behavior," Science, vol. 334, no. 6060, pp. 1269-1272, 2011.

[26] D. Centola and M. Macy, "Complex contagions and the weakness of long ties," American Journal of Sociology, vol. 113, no. 3, pp. 702-734, 2007.

[27] M. Zheng, L. Lü, M. Zhao et al., "Spreading in online social networks: The role of social reinforcement," Physical Review E, vol. 88, Article ID 012818, 2013.

[28] W. Wang, M. Tang, H.-F. Zhang, and Y.-C. Lai, "Dynamics of social contagions with memory of nonredundant information," Physical Review E, vol. 92, Article ID 012820, 2015. 
[29] J. Wu, M. Zheng, Z.-K. Zhang, W. Wang, C. Gu, and Z. Liu, "A model of spreading of sudden events on social networks," Chaos: An Interdisciplinary Journal of Nonlinear Science, vol. 28, Article ID 033113, 2018.

[30] J. Wu, M. Zheng, K. Xu, and C. Gu, "Effects of two channels on explosive information spreading," Nonlinear Dynamics, vol. 99, no. 3, pp. 2387-2397, 2020.

[31] J. Wu, M. Zheng, W. Wang, H. Yang, and C. Gu, "Double transition of information spreading in a two-layered network," Chaos: An Interdisciplinary Journal of Nonlinear Science, vol. 28, Article ID 083117, 2018.

[32] W. Wang, M. Tang, H.-F. Zhang, H. Gao, Y. Do, and Z.-H. Liu, "Epidemic spreading on complex networks with general degree and weight distributions," Physical Review E, vol. 90, Article ID 042803, 2014.

[33] P. Shu, Q.-H. Liu, S. Wang, and W. Wang, "Social contagions on interconnected networks of heterogeneous populations," Chaos: An Interdisciplinary Journal of Nonlinear Science, vol. 28, no. 11, p. 113114, 2018. 